\title{
Impacto da gestão estratégica de custos no desempenho econômico-financeiro
}

\author{
Impact of strategic management of costs on economic and financial performance
}

Impacto de la gestión estratégica de costos en el desempeño económico-financiero

\section{Marcelo Juarez Vizzotto}

Mestre em Administração pela Universidade de Caxias do Sul (UCS)

Professor dos Cursos de Ciências Contábeis, Administração e Gestão Comercial da Faculdade de Integração do Cone Sul (FISUL)

Endereço: Rua General Osório, 484/301. Bairro: São Bento.

CEP: 95703-150 - Bento Gonçalves/RS - Brasil

E-mail: marcelovizzotto.mv@gmail.com

Telefone: (54) 99163-4843

\section{Marta Elisete Ventura da Motta}

Doutora em Administração pela Pontifícia Universidade Católica do Rio Grande do Sul (PUC)

Professora da Universidade de Caxias do Sul (UCS)

Endereço: Rua Francisco Getúlio Vargas, 1130

CEP: 95070-560 - Caxias do Sul/RS - Brasil

E-mail: martamotta1234@gmail.com

Telefone: (54) 3218.2100

\section{Maria Emília Camargo}

Doutora em Engenharia de Produção na Universidade Federal de Santa Catarina (UFSC)

Professora do Programa de Pós-graduação em Administração e do Programa de Pós-graduação em Engenharia de Produção na Universidade de Caxias do Sul (UCS) e Pesquisadora da Unidade de Investigação Governança, Competitividade e Políticas Públicas (GOVCOPP) da Universidade de Aveiro - Portugal

Endereço: Av. João Machado Soares, 3199, Camobi

CEP: 97.110-000 - Santa Maria/RS - Brasil

E-mail: mariaemiliappga@gmail.com

Telefone: (55) 9-9972-8225

Artigo recebido em 28/03/2019. Revisado por pares em 06/10/2019. Reformulado em 11/12/2019. Recomendado para publicação em 20/03/2020 por Carlos Eduardo Facin Lavarda (Editor-Chefe). Publicado em 30/03/2020. 


\title{
Resumo
}

Quando a Gestão Estratégica de Custos (GEC) está alinhada com a estratégia da empresa as organizações tendem a apresentar um maior desempenho. A partir da relevância das questões relacionadas ao tema, esta pesquisa teve como objetivo analisar a relação existente entre as práticas da Gestão Estratégica de Custos e a atuação no mercado externo com o desempenho econômicofinanceiro nas empresas metalmecânicas, automotivas e eletroeletrônicas da Serra Gaúcha. Como procedimento metodológico, foi utilizada pesquisa exploratória, descritiva e quantitativa. $\mathrm{O}$ levantamento das informações foi realizado através de uma survey. A população foi composta por 367 empresas de pequeno, médio e grande porte. Obteve-se uma amostra de 87 empresas. Os dados foram analisados por meio da Análise Fatorial Exploratória e Modelagem de Equações Estruturais. Nas análises das hipóteses os resultados indicam a existência de relação significativa entre a utilização das práticas da GEC e o desempenho econômico-financeiro.

Palavras-chave: Gestão estratégica de custos; Desempenho econômico-financeiro; Empresas metalmecânicas, automotivas e eletroeletrônicas

\begin{abstract}
When Strategic Cost Management (SCM) is associated with the company's strategy, organizations tend to act better. However, from the relevance of the issues related to the topic, this research had the aim of analyzing the relationship between the practices of Strategic Cost Management and the performance in the foreign market with the economic-financial performance in the metalmechanic, automotive and electro-electronic companies of the Serra Gaúcha. As a methodological procedure, exploratory, descriptive and quantitative research was used. The information was collected through a survey. The population was made up of 367 small, medium and large companies. A sample of 87 companies was attained. The data were analyzed through Exploratory Factor Analysis and Modeling of Structural Equations. In the analysis of the hypotheses the results indicate the existence of a significant relationship between the use of SCM practices and economic-financial performance.
\end{abstract}

Keywords: Strategic cost management; Economic-financial performance; Metallurgical, automotive and electro-electronic enterprises

\section{Resumen}

Cuando la Gestión Estratégica de Costos (GEC) está alineada con la estrategia de la empresa, las organizaciones tienden a presentar un mayor desempeño. Con base en la relevancia de los temas relacionados con el tema, esta investigación tuvo como objetivo analizar la relación entre las prácticas de Gestión de Costos Estratégicos y el desempeño en el mercado extranjero con el desempeño económico-financiero de las empresas metalúrgicas, automotrices y electrónicas Serra Gaucha. Como procedimiento metodológico, se utilizó investigación exploratoria, descriptiva y cuantitativa. El levantamiento de las informaciones fue realizado a través de una encuesta. La población fue compuesta por 367 empresas de pequeño, mediano y grande porte. Se obtuvo una muestra de 87 empresas. Los datos fueron analizados por medio del Análisis Factorial Exploratorio y Modelado de Ecuaciones estructurales. En los análisis de las hipótesis los resultados indican la existencia de relación significativa entre la utilización de las prácticas de la GEC y el desempeño económico-financiero.

Palabras clave: Gestión estratégica de costos; Desempeño económico-financiero; Empresas metalmecánicas, automotrices y electro electrónicas

\section{Introdução}

Em meados do século XX a globalização proporcionou o aumento de competitividade entre as empresas e a inclusão de diversidade de produtos e processos nas organizações. Assim, a 
contabilidade existente já não oferecia informações relevantes para tomada de decisão, pois os procedimentos eram defasados e voltados para atender empresas do final do século XIX e início do século XX. Os quais não serviam para realização de planejamento e controle, pois eram informações de curto prazo e voltadas principalmente ao ciclo financeiro. (JOHNSON; KAPLAN, 1993).

Neste contexto, o tema Estratégia foi incorporado na contabilidade de gestão para responder as críticas sobre a perda de relevância das informações contábeis. (JOHNSON; KAPLAN, 1987). Assim, a gestão estratégica de custos surgiu da necessidade de atender as organizações inseridas em um contexto de produção de melhoria contínua da produtividade. (SHANK; GOVINDARAJAN, 1997). E sua atribuição passou a ser entendida como a geração de informações para suportar a tomada de decisão e subsidiar a formação da estratégia da empresa, permitindo assim, a otimização do desempenho organizacional. (BAINES; LANGFIELD-SMITH, 2003; ANDERSON, 2006).

Sob tal abordagem Alves (2012) relacionou práticas da gestão estratégica de custos com desempenho, sendo que objetivou relacionar o grau de evolução estratégica da gestão de custos na área de compras de empresas brasileiras e o desempenho financeiro obtido. Apesar da sua importância, Cadez e Guilding (2012) destacam que ainda há uma escassez de pesquisas empíricas sobre esta temática, o que evidencia a necessidade de se realizarem pesquisa para verificar se a utilização da gestão estratégica de custos alinhada com a estratégia organizacional está associada com um maior desempenho. Do mesmo modo, Wrubel (2009) complementa denotando a necessidade de se pesquisar as práticas de gestão estratégicas de custos que são adotadas pelas empresas e relacionar estas práticas com o desempenho das organizações.

Ademais, no Brasil, a partir da década de 90, com abertura do mercado externo, o ambiente de competitividade foi ampliado. Com isso, a Gestão Estratégica de Custos está sendo utilizada para integrar o processo de gestão de custos com o processo de gestão da empresa. (MARTINS, 2018). Neste contexto o objetivo deste estudo foi analisar a relação existentes entre as práticas da gestão estratégica de custos e a atuação no mercado externo com o desempenho econômicofinanceiro nas empresas metalmecânicas, automotivas e eletroeletrônicas da Serra Gaúcha.

As organizações devem manter a gestão estratégica de custos, objetivando conquistar espaço no mercado interno e externo, visto que o nível de concorrência está acirrado (RIBEIRO, 2010). Shank e Govindarajan (1997) concordam com esta necessidade, pois, de acordo com os autores, as informações da Gestão Estratégica de Custos são utilizadas para desenvolver estratégias e têm como objetivo a obtenção de vantagem competitiva. Assim, este trabalho busca contribuir com a identificação de quais práticas da GEC efetivamente possibilitam que as empresas apresentem maior desempenho econômico-financeiro e, ainda, indicar se atuar no mercado externo faz com que a empresa tenha melhor resultado.

\section{Referencial Teórico}

\subsection{Gestão estratégia de custos}

A partir da segunda metade dos anos 70, conforme explica Nakagawa (1991), emergiram novas filosofias de gestão empresarial. E foi nos anos 80 que as empresas deram espaço para implementação destas novas tecnologias de produção. Neste ambiente surgiu a necessidade de integração entre processos de controle gerencial e de gestão de manufaturas. Diante deste novo desafio, Johnson e Kaplan (1993) relatam que a contabilidade de gestão estava perdendo relevância, pois não fazia esta integração, ou seja, não havia conexão entre o processo produtivo e o controle gerencial. Para suprir esta lacuna, o tema estratégia foi integrado pela contabilidade de gestão.

Simmonds (1981) foi o primeiro a utilizar o termo Strategic Management Accounting 
(SMA) - Contabilidade de Gestão Estratégica - em uma publicação no Reino Unido. (GUILDING; CRAVENS; TAYLES, 2000; CADES; GUILDING, 2007; LANGFIELD-SMITH, 2008). Simmonds (1981) definiu SMA como o fornecimento e análise de dados de contabilidade de gestão sobre a empresa e seus concorrentes para utilização no desenvolvimento e acompanhamento de estratégia de negócio.

Através de Shank e Govindarajan, no ano de 1988, a contabilidade de gestão foi associada com gestão estratégica surgindo então a expressão Gestão Estratégica de Custos (LANGFIELDSMITH, 2008), integrando assim, as novas tecnologias de gestão com as de mensuração de custos (NAKAGAWA, 1991). Shank e Govindarajan (1997, p.4) definiram Gestão Estratégica de Custos como uma "análise de custos vista sob um contexto mais amplo, em que os elementos estratégicos se tornam mais conscientes, explícitos e formais. Aqui, os dados de custos são usados para desenvolver estratégias superiores a fim de se obter uma vantagem competitiva sustentável". No Brasil, coube a Nakagawa (1991) a divulgação do tema Gestão Estratégica de Custos, o qual entende que esta fornece informações para apoiar as decisões estratégicas e operacionais, utilizando os recursos produtivos de forma eficiente e eficaz.

Para Langfield-Smith (2008) existe uma variedade de técnicas incluídas sob o guardachuva da gestão estratégica de custos. Ribeiro (2010) destaca que algumas práticas já são utilizadas há décadas, mas de uma forma esparsa e não integrada. Para Cadez e Guilding (2007), apesar de haver um consenso limitado sobre o que constitui um quadro conceitual, não há uma lista definitiva sobre tais práticas.

Realizou-se uma pesquisa na base de dados da Scopus e também no Banco Digital de Dissertações e Teses (BDTD) para avaliar as pesquisas nesta temática que verificaram a utilização de práticas da Gestão Estratégica de Custos. Após uma análise dos trabalhos já realizados optouse por utilizar, nesta pesquisa, as práticas de gestão estratégica de custos do estudo de Rasia (2011), pois não existe uma relação única, ou seja, uma lista definitiva das práticas conforme explicaram Cadez e Guilding (2017) e pela autora ter realizado uma pesquisa sobre a utilização destas práticas.

As práticas analisadas no presente estudo, Quadro 1, conforme Rasia (2011), são divididas em 3 fatores: Melhoria dos Processos (a fim de atingir níveis elevados de qualidade aos menores custos possíveis), Redução de Custos (busca da redução de custos) e Desempenho em relação à dimensão do investimento (para avaliar o desempenho e analisar os investimentos realizados na estrutura e no desenvolvimento das operações).

\section{Quadro 1 - Os fatores e as práticas da GEC}

\begin{tabular}{|c|c|}
\hline Fatores & Práticas \\
\hline \multirow{5}{*}{ Melhoria dos Processos } & Custo Kaizen \\
\hline & Custo ambiental \\
\hline & Custo Total de Propriedade TCO \\
\hline & Gestão Baseada em Atividades ABM \\
\hline & Custo de ciclo de vida dos produtos \\
\hline & Análise Externa dos Custos \\
\hline \multirow{4}{*}{ Redução de Custos } & Custo-meta \\
\hline & Custos Intangíveis \\
\hline & Análise da cadeia de valor \\
\hline & Custo Padrão \\
\hline \multirow{3}{*}{$\begin{array}{l}\text { Desempenho em relação à dimensão } \\
\text { investimentos }\end{array}$} & Análise dos fatores determinantes de custo \\
\hline & Indicadores e métricas não financeiras \\
\hline & Custos logísticos \\
\hline
\end{tabular}

Fonte: Elaborado pelos autores com base em Rasia (2011).

Cada prática da GEC possui uma característica marcante apresentada no Quadro 2. 


\section{Quadro 2 - Característica das práticas da GEC}

\begin{tabular}{|c|c|}
\hline Prática & Principal Característica \\
\hline Custo Kaizen & $\begin{array}{l}\text { É a busca de soluções eficazes na área de melhoria de processo e consequente controle de } \\
\text { qualidade proporcionando menor custo (GOLAS et al., 2016). }\end{array}$ \\
\hline Custo ambiental & $\begin{array}{l}\text { Gastos necessários para implantar e operar o sistema de gestão e controle ambiental } \\
\text { (PADOVEZE, 2013). }\end{array}$ \\
\hline $\begin{array}{l}\text { Custo Total de } \\
\text { Propriedade TCO }\end{array}$ & $\begin{array}{c}\text { O Custo Total de Propriedade (representado pela sigla TCO, do inglês total cost of } \\
\text { ownership) é a soma de todos os gastos associados com a compra e utilização de um } \\
\text { produto ou serviço. Além do preço da compra podem ser incluídos gastos com transporte } \\
\text { a armazenamento, manutenção, operacionais, ambientais, saúde e segurança e da } \\
\text { eliminação (ELLRAM; SIFERD, 1993). }\end{array}$ \\
\hline $\begin{array}{l}\text { Gestão Baseada em } \\
\text { Atividades ABM }\end{array}$ & $\begin{array}{c}\text { Avalia o desempenho das atividades, a eficiência e a produtividade da empresa } \\
\text { (PADOVEZE, 2013). }\end{array}$ \\
\hline $\begin{array}{l}\text { Custo de ciclo de } \\
\text { vida dos produtos }\end{array}$ & $\begin{array}{l}\text { Fornece informações de custos desde a etapa do design do produto, estudo da viabilidade, } \\
\text { projeto de engenharia, desenvolvimento, fabricação, comercialização, distribuição, } \\
\text { manutenção até a retirada do produto do mercado e seu descarte (ATKINSON, et al., } \\
\text { 2000; WERNEKE, 2001; PEREZ Jr; OLIVEIRA; COSTA, 2012). }\end{array}$ \\
\hline $\begin{array}{l}\text { Análise Externa dos } \\
\text { Custos }\end{array}$ & $\begin{array}{c}\text { Para Bartz, Staudt e Souza (2005), a análise dos custos dos concorrentes, do qual a } \\
\text { organização disputa mercado, complementa a análise de seus custos internos. Saber quais } \\
\text { são os custos dos concorrentes ajuda a empresa na tomada de suas decisões e na } \\
\text { formulação de suas estratégias. Kotler (2000) informa que cada setor organizacional } \\
\text { possui determinada composição de custos e isso é o que irá moldar, em parte, a sua } \\
\text { estratégia. Assim, a análise externa dos custos permite que a empresa se posicione } \\
\text { estrategicamente (ROCHA, 1999). }\end{array}$ \\
\hline Custo-meta & $\begin{array}{l}\text { Estabelecimento de um custo-alvo para o produto na fase de seu planejamento e desenho } \\
\text { (SAKURAI, 1997). }\end{array}$ \\
\hline Custos Intangíveis & $\begin{array}{l}\text { A parcela de sacrifício financeiro absorvida na formação ou manutenção de um fator } \\
\text { intangível (DIEHL, 1997). }\end{array}$ \\
\hline $\begin{array}{l}\text { Análise da cadeia de } \\
\text { valor }\end{array}$ & $\begin{array}{l}\text { Conjunto de atividades interempresas no sentido de criar valor desde a compra de } \\
\text { matérias-primas até o atendimento ao cliente final (COKINS, 2001). }\end{array}$ \\
\hline Custo Padrão & $\begin{array}{l}\text { Acompanhamento do custo do produto durante o processo produtivo que permite avaliar } \\
\text { se o que foi pré-determinado está sendo executado (BOGDANOIU, 2013). }\end{array}$ \\
\hline $\begin{array}{l}\text { Análise dos fatores } \\
\text { determinantes de } \\
\text { custo }\end{array}$ & $\begin{array}{l}\text { Análise dos fatores que determinam as causas que geraram os custos para a organização } \\
\text { (SHANK; GOVINDARAJAN, 1997). }\end{array}$ \\
\hline $\begin{array}{l}\text { Indicadores e } \\
\text { métricas não } \\
\text { financeiras }\end{array}$ & $\begin{array}{l}\text { Permitem analisar os fatores que provocam o desempenho da organização (SHANK; } \\
\text { GOVINDARAJAN, 1997). }\end{array}$ \\
\hline Custos logísticos & $\begin{array}{l}\text { São os custos gerados pela movimentação do inventário, desde a entrada até o ponto de } \\
\text { consumo (MARTINS, 2018). }\end{array}$ \\
\hline
\end{tabular}

Fonte: Elaborado pelos autores (2019).

\subsection{Desempenho}

A análise do desempenho possibilita auxiliar na prevenção de incertezas do ambiente organizacional reduzindo a tentativa dos gestores em realizar investimentos de capital, principalmente em empresas que atuam em ambiente de elevado risco tecnológico e econômico (CHOW; COOPER; HADDAD, 1991). Ferramentas gerenciais como o Balanced Scorecard chamam a atenção para a multidimensionalidade do desempenho organizacional (MATITZ; BULGACOV, 2011). Neste contexto, Azeredo, Souza e Machado (2009) afirmam que a avaliação de desempenho permite à empresa uma análise comparativa entre o que foi planejado e efetivamente os resultados obtidos.

$\mathrm{Na}$ avaliação de desempenho, podem ser utilizados indicadores financeiros e não financeiros. Os indicadores não financeiros procuram avaliar aspectos intangíveis, não encontradas em demonstrações contábeis, como os relacionados com as expectativas de clientes, fornecedores, concorrentes e recursos humanos (KAPLAN; NORTON, 2000). Já os indicadores financeiros têm 
como fonte a contabilidade financeira que, conforme Perez Junior e Begalli (1999) e Silva (2005), caracteriza-se como uma das melhores ferramentas para se avaliar desempenho seja no passado, presente e futuro de uma empresa para tomada de decisões. Gitman (2010) reforça que a análise de indicadores econômicos financeiros serve para comparar o desempenho da própria organização e também empresas do mesmo segmento em vários períodos distintos.

Conforme Assaf Neto (2015), por meio dos indicadores de desempenho econômicofinanceiro é possível medir o desempenho da empresa. Ademais, são fontes de subsídios ao gestor para tomada de decisão. Para o autor, por meio dos indicadores econômicos é possível medir o capital próprio da empresa com a rentabilidade da sua atividade operacional. Já por meio dos indicadores financeiros é possível avaliar a disponibilidade de caixa em relação às suas dívidas e sua estrutura de capital.

A avaliação do desempenho econômico, segundo Assaf Neto (2015), pode ser processada por meio da formulação do retorno sobre o investimento (ROI), do retorno sobre o ativo (ROA) e do retorno sobre o patrimônio líquido (ROE). E para avaliação do desempenho financeiro, de acordo com o mesmo autor, pode-se utilizar o EBITDA (termo em inglês que significa Earning Before Interest, Taxes, Depreciation/Depletion and Amortization, em português, lucro antes dos juros, impostos, depreciações/exaustões e amortizações), pois é o indicador financeiro globalizado. Equivale ao conceito de fluxo de caixa operacional apurado antes do cálculo do IR.

\subsection{Hipóteses e modelo teórico}

Segundo Rasia (2011) as práticas de GEC que fazem parte do fator Melhoria dos Processos contribuem para empresa atingir níveis elevados de qualidades com os menores custos possíveis. As práticas da GEC pertencente ao fator Redução de Custos buscam reduzir os custos nas organizações. E, as práticas que fazem parte do fator Desempenho em Relação aos Investimentos visam avaliar o desempenho e analisar os investimentos realizados na estrutura e no desenvolvimento das operações.

Considerando ainda que, a utilização de práticas da GEC, de acordo com Baines e Langfield-Smith (2003), Anderson (2006), Dieh (2006) Henri, Boiral e Roy (2006) e Cadez e Guilding (2012), contribui para que a organização aumente seu desempenho, apresentam-se as seguintes hipóteses.

H1. A utilização das práticas de gestão estratégica de custos, dimensão melhoria dos processos (DMP), influencia positivamente no desempenho econômico-financeiro (DEF).

H2. A utilização das práticas de gestão estratégica de custos, dimensão redução de custos (DDR), influencia positivamente no desempenho econômico-financeiro (DEF).

H3. A utilização das práticas de gestão estratégica de custos, dimensão desempenho em relação aos investimentos (DDI), influencia positivamente no desempenho econômico-financeiro (DEF).

Uma organização, segundo Alotaibi e Zhang (2016), que visa o mercado externo, tem impacto positivo em seu desempenho. Os autores testaram empiricamente esta relação e comprovaram que a mesma é significativa. Afirmam, por fim que os investimentos voltados para exportação geram maior desempenho. Estudos como o de Naudé e Rossouw (2011) evidenciam que as exportações são capazes de alavancar economia de países. Em seus estudos evidenciaram essa afirmação comparando o desempenho de países Brasil, China, Índia e África do Sul. Assim, propõe-se a quarta hipótese.

H4. A atuação no mercado externo influencia positivamente no desempenho econômicofinanceiro (DEF).

Com base nas hipóteses da pesquisa, para facilitar a compreensão entre os construtos, elaborou-se o modelo teórico proposto, conforme apresentado na Figura 1, onde DMP significa 
Melhoria nos Processos; DDI significa Desempenho em Relação aos Investimentos; DDR dignifica Redução de Custos; e, DEF significa Desempenho Econômico-Financeiro.

Figura 1 - Modelo Teórico Proposto

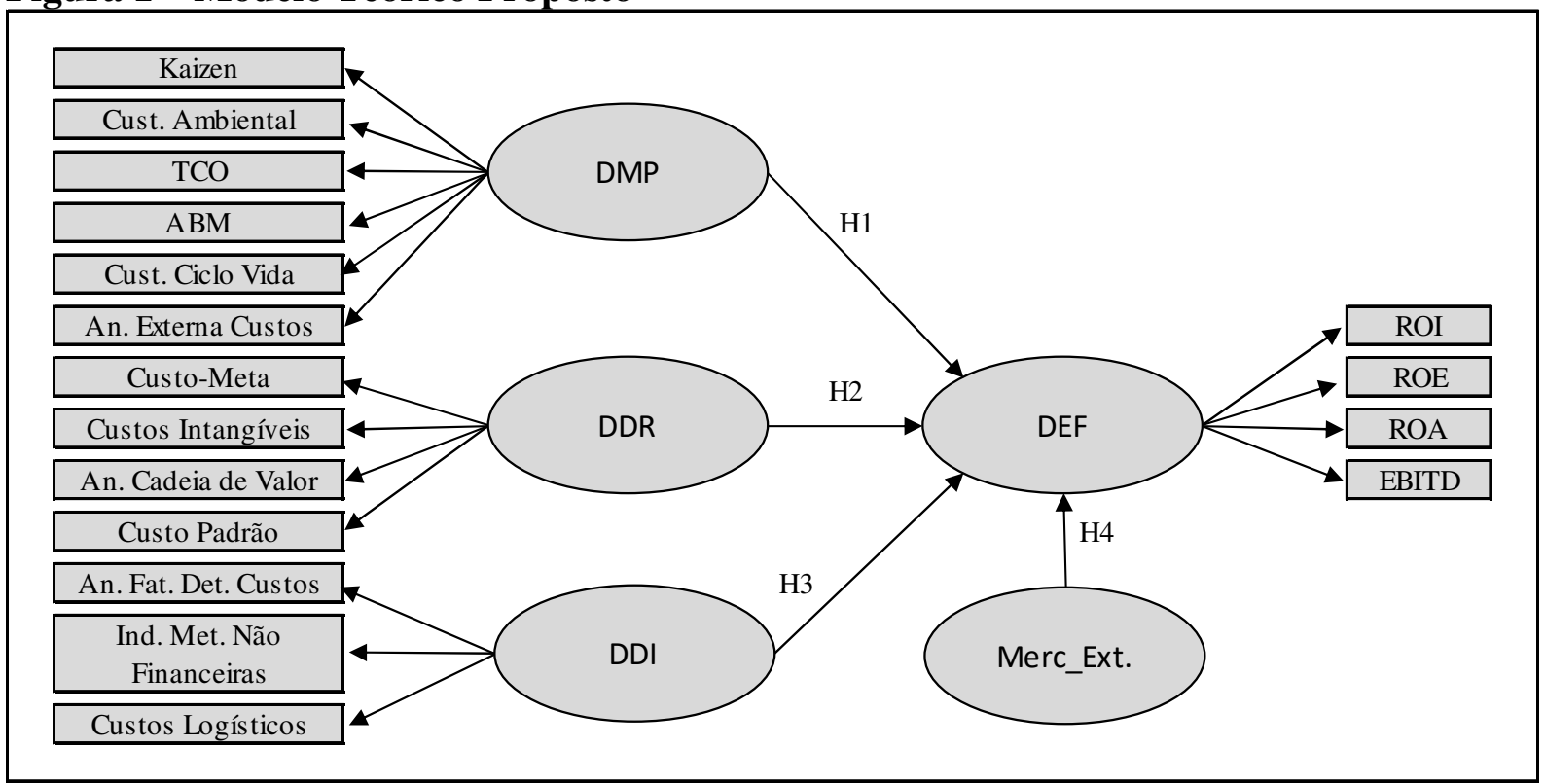

Fonte: Elaborado pelos autores (2019).

\section{Método}

Este estudo adotou a estratégia metodológica do tipo quantitativo-descritiva, cuja investigação empírica ocorreu através de uma survey e cuja aplicação ocorreu por meio de formulário eletrônico. (GIL, 2008).

$\mathrm{O}$ instrumento de coleta de dados utilizado continha 3 blocos. $\mathrm{O}$ primeiro bloco de questões, com 13 variáveis adaptadas de Rasia (2011) teve por finalidade verificar a utilização das práticas de gestão estratégica de custos pelas empresas. As respostas foram registradas em uma escala do tipo likert de 5 pontos, sendo 1 raramente utiliza e 5 utiliza sempre. O segundo bloco de questões pretendeu avaliar o desempenho econômico-financeiro das empresas. Foi adaptado de Almeida (2010) e conta com quatro variáveis (indicadores). As respostas foram registradas em uma escala do tipo likert de 5 pontos, sendo 1 muito menor e 5 muito maior. Já o terceiro bloco identificou o perfil dos respondentes e da empresa.

A coleta de dados foi realizada a partir de questionário eletrônico enviado a funcionários ligados à área de custos. Portanto, a população deste estudo era composta por 367 indústrias de pequeno, médio e grande porte dos setores metalomecânico, automotivo e eletroeletrônico da Serra Gaúcha filiadas ao SIMECS. Das quais obteve-se um retorno de 90 empresas, as quais formaram a amostra da pesquisa. O número de respondentes encontra-se adequado de acordo com os pressupostos estabelecidos por Hair Jr et al. (2009) em que o número mínimo de respondente por variável deve ser de no mínimo 05 (cinco). Assim, considerando que o número de variáveis (17) do instrumento de coleta a amostra mínima exigida era de 85 (oitenta e cinco) respondentes.

A preparação dos dados é o passo inicial em que se avalia o impacto dos dados perdidos, se identifica observações atípicas e testas as suposições inerentes à maioria das técnicas multivariadas. (HAIR Jr. et al., 2009). Seguindo as orientações de Hair Jr. et al. (2005) 3 repostas foram descartadas, uma por estar incompleta, outra por não pertencer a população e uma última por ter respondido em duplicidade. Assim, dos 90 (noventa) retornos 87 (oitenta e sete) permaneceram válidos. Em um segundo momento, com o auxílio do SPSS, analisou-se as 
frequências para identificar a existência de outliers, onde não foram encontrados resultados atípicos que justificassem a exclusão de algum participante (HAIR et al., 2005).

Por conseguinte, realizaram-se os testes de normalidade, homocedasticidade, linearidade e multicolinearidade, pois de acordo com Hair Jr. et al. (2009) são suposições que afetam a estatística univariada e multivariada. Os dados da pesquisa apresentaram normalidade, por meio da realização dos testes de assimetria e curtose, estando os resultados de assimetria entre -3 a +3 e curtose entre - 10 a + 10 (HAIR Jr. et al., 2005; HAIR Jr et al., 2009). Para verificação da homocedasticidade Hair Jr. et al. (2009) recomenda a utilização do teste de Levene. Neste estudo considerou-se a variável dependente a variável Métodos de Custeio, considerada não métrica $(1=$ Método de Custeio por Absorção; 2 = Método de Custeio Baseado em Atividades e 3 = Método de Custeio Variável) que foi analisada com as demais variáveis independentes métricas do instrumento. Observou-se que somente a variável C_Ambiental apresentou significância menor do que 0,05 no teste de homogeneidade de variância, sugerindo assim heteroscedasticidade da variância. Apesar disso, esta variável não foi excluída da análise, pois conforme de Hair Jr. et al. (2009), uma variável em um total de 17 não indicam implicações significativas nas análises futuras.

A linearidade desta pesquisa foi realizada por meio da existência de relações lineares entre as variáveis dos construtos. Hair Jr. et al. (2009) recomenda, para determinar a correlação existente, a análise de correlação bivariada por meio do coeficiente de Pearson. De acordo com Hair Jr. et al. (2009) e Kline (2011) o coeficiente de correlação de Pearson pode variar entre -1 e +1 . Assim, quanto mais próximos de -1 ou +1 maior é o grau de associação entre as variáveis indicando a presença de uma relação linear.

A análise da multicolinearidade, de acordo com Fornell e Bookstein (1982) e Hair Jr. et al. (2009), deve ser feita por meio dos testes de Variance Inflation Factor (VIF) e Valor de Tolerância. Os parâmetros para estes testes são de até 5 para o VIF e entre 0,2 e 0,8 para o Valor de Tolerância (FORNELL; BOOKSTEIN, 1982; HAIR Jr. et. al., 2009). Os resultados do teste de multicolinearidade desta pesquisa ficaram dentro dos parâmetros estabelecidos pela literatura.

Nesta pesquisa, buscando responder ao objetivo proposto, adotou-se a estratégia de desenvolvimento de modelos, analisados a partir da Modelagem de Equações Estruturais (MEE). (HAIR. et al., 2009; BYRNE, 2010; KLINE, 2011).

\section{Análise dos Dados}

A pesquisa foi aplicada nas empresas de pequeno, médio e grande portes filiadas ao SIMECS. A amostra foi de 87 empresas, sendo que a grande parcela dos respondentes $62,1 \%$ (54 empresas) são de pequeno porte. A localização geográfica predominantemente foi no município de Caxias do Sul com 69\% (60 empresas) dos respondentes. Uma característica marcante das empresas participantes é o seu tempo de atuação no mercado, sendo que 95,4\% (83 empresas) possuem acima de 11 anos de atuação.

O setor participante foi predominantemente o metalmecânico com 77\% (67 empresas) dos respondentes. O faturamento das empresas em sua maioria 55,4\% (48 empresas) supera os $\mathrm{R} \$$ 12.000.000,00 anuais e a exportação está presente em 60 empresas participantes do estudo. Como característica quanto ao cálculo de custos o método de custeio por absorção é predominante, sendo utilizado por 58,6\% (51 empresas) dos respondentes. Quanto aos respondentes percebe-se que esta pesquisa conseguiu direcionar o questionário aos responsáveis pelo controle de custos das empresas, sendo que 51 respostas foram retornadas por gestores de custos ou gerentes e supervisores. Os respondentes apresentam experiência em sua função e 79 deles afirmam possuir graduação. 


\subsection{Confiabilidade}

Para verificar consistência interna realizou-se a verificação através do Alfa de Cronbach, sendo que o limite aceito é de 0,7 . Neste estudo, as 17 variáveis apresentaram um valor para este teste de 0,875 demonstrando que o instrumento é fidedigno. Adicionalmente, para verificar se o método de análise fatorial é adequado para o tratamento dos dados realizou-se o teste de Medida de Adequação da Amostra de Kaiser Meyer Olkin (KMO) e o Teste de Esfericidade de Bartlett que são os testes indicados por Hair Jr. et al. (2009). Segundo estes autores o teste KMO deve ter como resultado mínimo 0,70 enquanto que o teste de Esfericidade de Bartlett deve ter sig. $<0,05$.

Os resultados da pesquisa demonstram um KMO de 0,840 que Hair Jr. et al. (2009) classificam como admirável. E o nível de significância do teste de Esfericidade de Bartlett foi de 0,000 dentro dos parâmetros estabelecidos. Desse modo, os resultados encontrados demonstram que a matriz de dados possui, com base em Hair Jr. et al. (2009), correlações suficientes para justificar a aplicação da análise fatorial.

\subsection{Unidimensionalidade}

A unidimensionalidade tem como objetivo identificar qual fator a variável pertence, ou seja, verificar quais relações existem entre as variáveis propostas. $\mathrm{O}$ procedimento adotado para testar a unidimensionalidade é a análise fatorial exploratória. Por meio desta análise é possível compreender quais variáveis podem atuar juntas e quais delas tem impacto na análise, ou seja, é possível avaliar a relação entre as variáveis (HAIR JR. et al., 2009).

A comunalidade, de acordo com Hair Jr. et al. (2009), explica o quanto uma variável se correlaciona com uma ou mais variáveis. Relatam estes autores que esse relacionamento pode ser medido por um índice que varia de 0 a 1 , sendo que quando o resultado for abaixo de 0,5 a variável pode ser omitida ou eliminada. A Tabela 1 apresenta os resultados desta análise.

Tabela 1 - Comunalidade das variáveis

\begin{tabular}{|c|c|c|}
\hline \multicolumn{3}{|c|}{ Comunalidades } \\
\hline & Inicial & Extração \\
\hline Kaizen & 1,000 & 0,698 \\
\hline C_Ambiental (Custo Ambiental) & 1,000 & 0,377 \\
\hline TCO (Custo Total de Propriedade) & 1,000 & 0,666 \\
\hline ABM (Gestão Baseada em Atividades) & 1,000 & 0,512 \\
\hline C_Ciclo_Vida (Custo do Ciclo de Vida) & 1,000 & 0,712 \\
\hline An_Ext_Cust (Análise Externa dos Custos) & 1,000 & 0,667 \\
\hline Custo_Meta & 1,000 & 0,548 \\
\hline Custo_Intang (Custo Intagível) & 1,000 & 0,630 \\
\hline An_Cad_Valor (Análise da Cadeia de Valor) & 1,000 & 0,659 \\
\hline Custo_Padrão & 1,000 & 0,516 \\
\hline An_Fat_Det (Análise dos Fatores Determinantes) & 1,000 & 0,528 \\
\hline Ind_Met_NF (Indicadores e Métricas não Financeiras) & 1,000 & 0,464 \\
\hline Custos_Log (Custos Logísticos) & 1,000 & 0,732 \\
\hline ROE (Retorno sobre o Patrimônio Líquido) & 1,000 & 0,895 \\
\hline ROI (Retorno sobre o Investimento) & 1,000 & 0,874 \\
\hline ROA (Retorno sobre o Ativo) & 1,000 & 0,935 \\
\hline $\begin{array}{l}\text { EBITDA (lucro antes dos juros, impostos, } \\
\text { depreciações/exaustões e amortizações }\end{array}$ & 1,000 & 0,753 \\
\hline
\end{tabular}

Método de extração: análise do componente principal.

Fonte: elaborado pelos autores (2019).

Embora as variáveis C_Ambiental e Ind_Met_NF tenham apresentado comunalidades menores do que 0,5 , e consequentemente não ter um relacionamento razoável com as outras 
variáveis, elas não foram excluídas da análise fatorial exploratória, pois as variáveis são importantes para o objetivo do estudo (HAIR JR. et al., 2009).

Tabela 2 - Método de extração dos principais componentes

\begin{tabular}{|c|c|c|c|c|c|c|c|c|c|}
\hline \multicolumn{10}{|c|}{ Variação total explicada } \\
\hline \multirow{2}{*}{ Componente } & \multicolumn{3}{|c|}{ Valores próprios iniciais } & \multicolumn{3}{|c|}{$\begin{array}{c}\text { Somas de extração de } \\
\text { carregamentos ao quadrado }\end{array}$} & \multicolumn{3}{|c|}{$\begin{array}{c}\text { Somas rotativas de } \\
\text { carregamentos ao quadrado }\end{array}$} \\
\hline & Total & $\begin{array}{c}\% \text { de } \\
\text { variação }\end{array}$ & $\begin{array}{c}\% \\
\text { cumulativa }\end{array}$ & Total & $\begin{array}{c}\% \text { de } \\
\text { variação }\end{array}$ & $\begin{array}{c}\% \\
\text { cumulativa }\end{array}$ & Total & $\begin{array}{c}\% \text { de } \\
\text { variação }\end{array}$ & $\begin{array}{c}\% \\
\text { cumulativa }\end{array}$ \\
\hline 1 & 5,759 & 33,876 & 33,876 & 5,759 & 33,876 & 33,876 & 3,613 & 21,252 & 21,252 \\
\hline 2 & 3,143 & 18,486 & 52,362 & 3,143 & 18,486 & 52,362 & 2,718 & 15,991 & 37,243 \\
\hline 3 & 1,231 & 7,241 & 59,603 & 1,231 & 7,241 & 59,603 & 2,458 & 14,459 & 51,702 \\
\hline 4 & 1,032 & 6,070 & 65,673 & 1,032 & 6,070 & 65,673 & 2,375 & 13,972 & 65,673 \\
\hline 5 & 0,918 & 5,402 & 71,076 & & & & & & \\
\hline 6 & 0,786 & 4,623 & 75,699 & & & & & & \\
\hline 7 & 0,731 & 4,303 & 80,001 & & & & & & \\
\hline 8 & 0,602 & 3,541 & 83,542 & & & & & & \\
\hline 9 & 0,523 & 3,074 & 86,616 & & & & & & \\
\hline 10 & 0,484 & 2,847 & 89,464 & & & & & & \\
\hline 11 & 0,417 & 2,454 & 91,918 & & & & & & \\
\hline 12 & 0,371 & 2,184 & 94,102 & & & & & & \\
\hline 13 & 0,312 & 1,838 & 95,940 & & & & & & \\
\hline 14 & 0,308 & 1,811 & 97,751 & & & & & & \\
\hline 15 & 0,214 & 1,261 & 99,012 & & & & & & \\
\hline 16 & 0,113 & 0,664 & 99,676 & & & & & & \\
\hline 17 & 0,055 & 0,324 & 100,000 & & & & & & \\
\hline
\end{tabular}

Fonte: elaborado pelos autores (2019).

Quanto a análise de extração de fatores (componentes), conforme Tabela 2, os fatores representam 65,673\% da variância das 17 variáveis, considerado suficiente em termos de variância total explicada. Hair Jr. et al. (2009) relatam que os fatores mantidos devem explicar pelo menos $60 \%$ da variância. Além disso, os 4 fatores possuem raízes latentes de autovalores maior que 1 que é o critério para ser considerado significante de acordo com o estabelecido por Hair Jr. et al. (2009).

Após determinar o número de fatores (componentes) gerou-se a matriz de fatores (componentes) para definir o agrupamento das variáveis. Na sequência empregou-se o método rotacional Varimax, determinado por Hair Jr. et al. (2009), a fim de melhorar a interpretação e redução de ambiguidades que acompanham as soluções fatoriais não rotacionais, eliminando, também, as cargas cruzadas.

Como resultado obteve-se a Matriz de Fatores (Componentes) conforme Tabela 3. O fator 1 foi denominado como desempenho econômico-financeiro. Os fatores 2, 3 e 4 foram nomeados com as mesmas denominações estabelecidas por Rasia (2011), quais sejam: desempenho em relação a dimensão do investimento, redução de custos e melhoria dos processos, respectivamente.

Assim, no fator 1, desempenho econômico-financeiro, ficaram agrupadas as variáveis ROA, ROE, ROI e EBITDA. As três primeiras representam os indicadores econômicos e a última $\mathrm{o}$ indicador financeiro.

O fator 2, denominado desempenho em relação ao investimento, reuniu as práticas custos logísticos, análise dos fatores determinantes de custos, custo padrão e custo ambiental. Este fator é explicado por variáveis que acompanham o custo do inventário, analisam os fatores que causam os custos, acompanham os custos durante o processo produtivo e controlam os gastos para gestão ambiental. 
Tabela 3 - Matriz de Fatores (componentes)

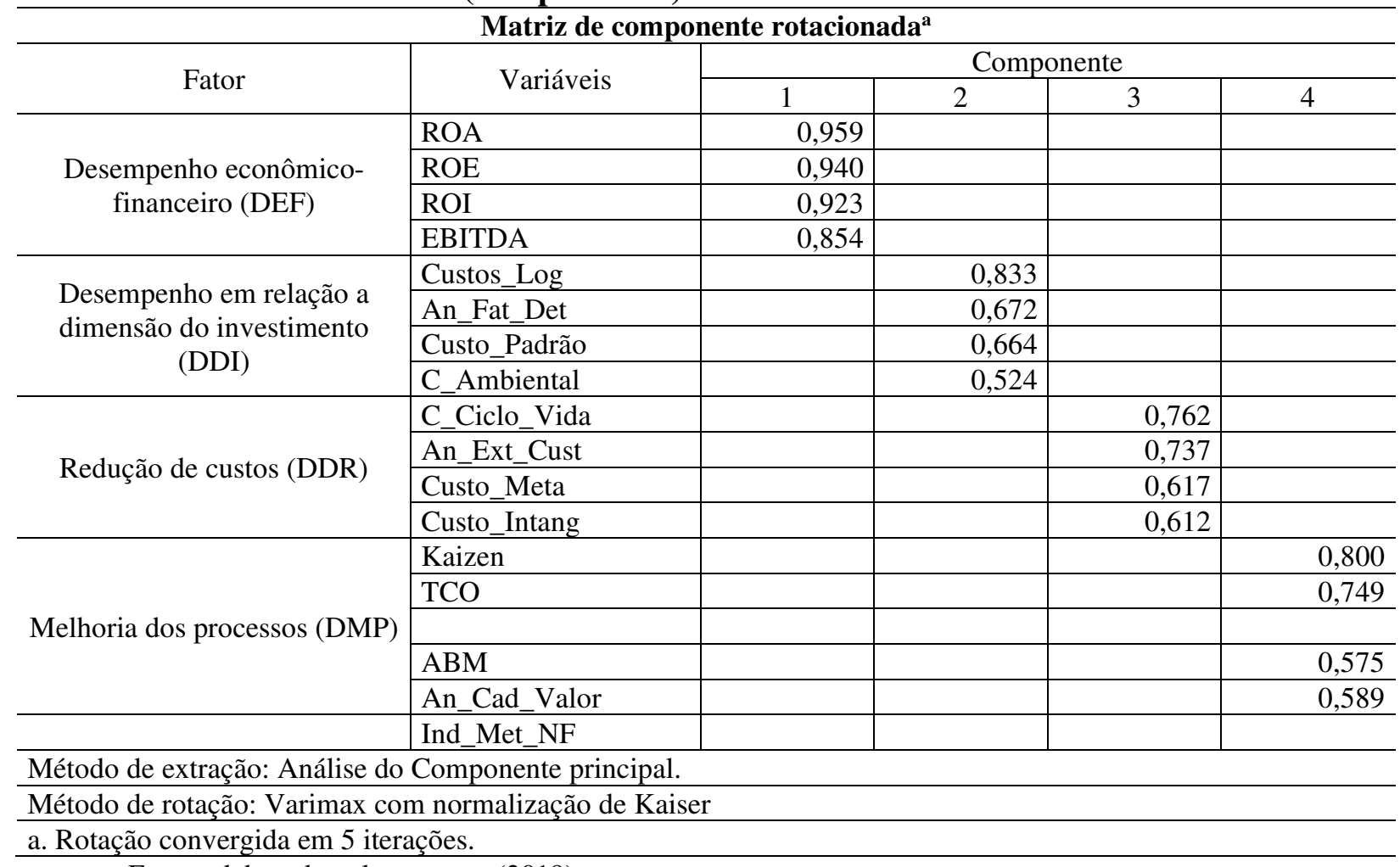

Fonte: elaborado pelos autores (2019).

O fator 3, redução de custos, conta com as práticas custo do ciclo de vida, análise externa de custos, custo meta e custos intangíveis. Estas práticas fornecem informações de custos: da etapa de design até o descarte do produto, dos custos dos concorrentes, dos custos estabelecidos como alvo no planejamento e desenho do produto e dos custos para formar ou manter um fator intangível.

O fator 4, melhoria dos processos, é representado pelo custo Kaizen, custo total de propriedade (TOC), gestão baseadas em atividades (ABM) e análise da cadeia de valor. Avaliam os custos para melhoria contínua, os gastos relacionados com a compra de um produto, o desempenho das atividades e a eficiência produtiva da empresa e os custos de todas as atividades da cadeia de valor.

Por fim, a prática de Gestão de Custos denominada indicadores e métricas não financeiras apresentou carga fatorial menor do que 0,50 e por este motivo foi eliminada (HAIR JR. et al., 2009).

\subsection{Validade Convergente}

Após garantir que uma escala está de acordo com sua definição conceitual, que é unidimencional e que atende aos níveis de confiabilidade é necessário fazer uma avaliação final que é a validade de escala, a qual representa o grau que uma escala ou conjunto de medidas representa o conceito de interesse. A validade pode ser convergente e discriminante (HAIR JR. et al., 2009).

De acordo com Hair Jr. et al. (2009) e Marôco (2010) a validade convergente avalia o grau em que duas medidas do mesmo conceito estão correlacionadas, sendo que correlações altas indicam que a escala está medindo o conceito pretendido. Para mediar a validade convergente analisa-se a confiabilidade composta, na qual é considerado adequado o índice $\geq 0,7$; a variância extraída deve ter índice $\geq 0,5$; e a confiabilidade que deve ter uma carga $\geq 0,6$, sendo maior do que 0,7 um bom valor. 
Na primeira etapa, os quatro construtos foram analisados através do software AMOS $®$, com o objetivo de obter os coeficientes de regressão padronizados. Assim com os coeficientes de regressão padronizados, foi realizado o cálculo da Confiança Composta $(\mathrm{CC})$ e da Análise de Variância Extraída (AVE), para cada um dos construtos. Os construtos considerados são: DMP, DDR, DDI e DEF. Foi utilizado o software MS-Excel® para o cálculo da CC e da AVE dos fatores (com base nos Coeficientes de Regressão Padronizados). Os resultados obtidos ficaram dentro do estabelecido pela literatura, ou seja, apresentam validade convergente.

\subsection{Validade Discriminante}

De acordo com Hair Jr. et al. (2009) e Marôco (2010) a validade discriminante demonstra o quanto os construtos investigados estão correlacionados, bem como o quanto diferem entre si. Para efetividade dos testes de Validade Discriminante, este estudo utilizou os preceitos de Fornell e Larcker (1981) que comparam as variâncias extraídas dos construtos com as variâncias compartilhadas, as quais são obtidas a partir das correlações entre construtos elevados ao quadrado.

Fornell e Larcker (1981) sugerem que a variância média extraída (AVE) seja comparada com o quadrado da correlação das variáveis latentes, sendo que a variância média extraída deve apresentar valore igual ou superior do que as respectivas variâncias compartilhadas. A Tabela 4 apresenta a matriz para a análise da validade discriminante, com a diagonal principal contendo a AVE para cada construto e as demais células apresentando o quadrado dos coeficientes de correlação entre cada par de construtos.

Segundo dados da Tabela 4, observa-se que todas as variâncias compartilhadas são inferiores à variância extraída pelos itens que medem os construtos, indicando validade discriminante adequada.

Tabela 4 - Validade Discriminante pelo Critério de Fornell e Larcker (1981)

\begin{tabular}{l|l|l|l|l}
\hline & DMP & DDR & DDI & DEF \\
\hline DMP & $\mathbf{0 , 4 8 4 7}$ & & & \\
\hline DDR & 0,3612 & $\mathbf{0 , 4 3 4 0}$ & & \\
\hline DDI & 0,3375 & 0,3147 & $\mathbf{0 , 4 1 6 3}$ & \\
\hline DEF & 0,0501 & 0,0187 & 0,0396 & $\mathbf{0 , 8 2 4 0}$ \\
\hline
\end{tabular}

Fonte: elaborado pelos autores (2019)

Contudo, verificando todos os resultados apresentados para a análise fatorial confirmatória, julga-se que o modelo de mensuração proposto atende os requisitos desejados de confiabilidade, unidimensionalidade, validade convergente e validade discriminante, sendo, portanto, possível o estudo por meio de um modelo estrutural.

\subsection{Validação do modelo teórico}

\subsubsection{Ajuste do modelo teórico}

A validação do Modelo Teórico foi realizada conforme definido nos aspectos metodológicos desta pesquisa por meio das medidas de ajuste do modelo (HAIR JR. et al., 2009; BYRNE, 2010; KLINE, 2011). Assim, o modelo teórico em que serão verificadas as medidas de ajustes para validação está representado na Figura 2. Importante ressalvar que para construir este modelo outros foram testados. 
Figura 2 - Modelo construído em função dos resultados da Análise Fatorial Exploratória

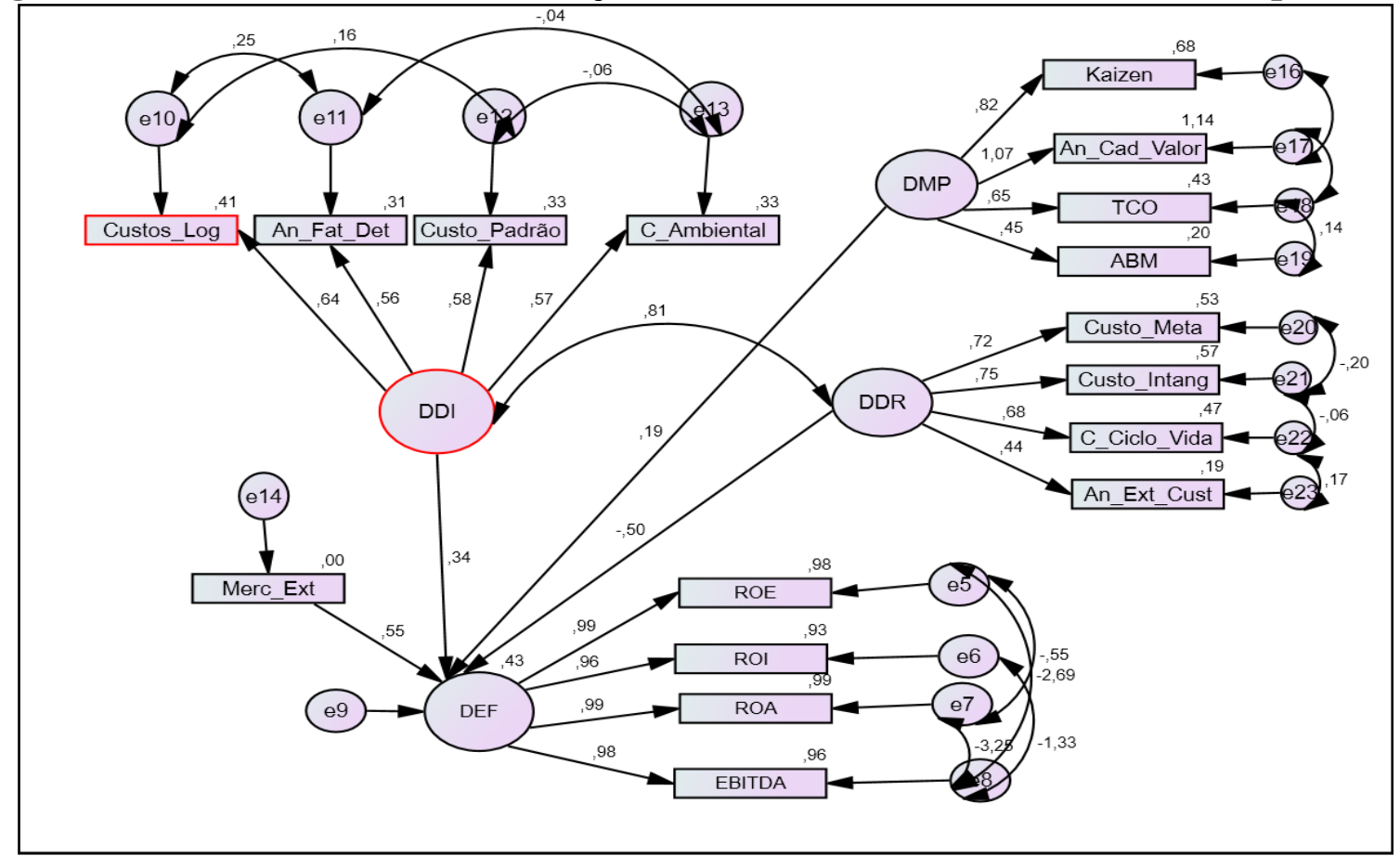

Fonte: elaborado pelos autores (2019)

Para avaliar o modelo desta pesquisa foram realizados testes para verificar a medida de ajuste absoluto, incremental e parcimonioso. Os índices resultantes estão dispostos na Tabela 5. Pode-se observar que os índices resultantes estão dentro dos parâmetros das medidas de ajustes indicadas pela literatura.

Tabela 5 - Medidas de ajustes do Modelo Teórico

\begin{tabular}{c|c|c}
\hline Categorias & Medidas de Ajuste & Índices \\
\hline \multirow{3}{*}{ Absoluto } & $\mathrm{x}^{2} / \mathrm{gl}$ & 1,152 \\
\cline { 2 - 3 } & GFI & 0,994 \\
\cline { 2 - 3 } & AGFI & 0,975 \\
\cline { 2 - 3 } & RMSEA & 0,010 \\
\hline \multirow{2}{*}{ Incremental } & TLI & 1,034 \\
\cline { 2 - 3 } & NFI & 0,990 \\
\hline Parcimonioso & CFI & 1,000 \\
\hline
\end{tabular}

Fonte: elaborado pelos autores (2019).

O Chi-Quadrado foi de 1,152 quando o indicado é menor do que 3. O GFI resultante foi de 0,994 e o AGFI foi de 0,975, para ambos o recomendado é que tenham índices maiores de 0,90 para ser considerado um bom ajuste do modelo. Já o RMSEA foi de 0,010 demonstrando um ajuste perfeito, pois é menor do que 0,03 .

Quanto a medida incremental o TLI foi de 1,034 e o NFI foi de 0,990 ambos com valores acima de 0,90 apontando um bom ajuste do modelo. Por fim, a medida CFI, apresentou um índice de 1,000 também dentro dos parâmetros aceitáveis, ou seja, superior a 0,90.

\subsubsection{Teste de hipóteses}

Na sequência, dando continuidade à validação do modelo, realizou-se o teste de hipóteses. Examinou-se a significância e a magnitude dos coeficientes de regressão estimado. O coeficiente de regressão mede a quantidade de mudança esperada na variável dependente para cada unidade 
de mudança da variável independente. O sinal deste coeficiente indica se a correlação é positiva ou negativa (HAIR JR. et al., 2009).

Na Tabela 6 estão dispostas as hipóteses propostas, os caminhos estruturais, os coeficientes não padronizados, os erros padrão, os coeficientes padronizados e os resultados do Modelo.

Tabela 6 - teste de Hipóteses do Modelo Teórico Proposto

\begin{tabular}{c|c|c|c|c|c|c}
\hline Hi & $\begin{array}{c}\text { Caminhos } \\
\text { Estruturais }\end{array}$ & $\begin{array}{c}\text { Coeficientes } \\
\text { Não } \\
\text { Padronizados } \\
(\mathbf{b})\end{array}$ & $\begin{array}{c}\text { Erro } \\
\text { Padrão }\end{array}$ & $\begin{array}{c}\text { Coeficientes } \\
\text { Padronizados } \\
(\boldsymbol{\beta})\end{array}$ & $\mathbf{p}$ & Resultados \\
\hline H1 & DMP $\rightarrow$ DEF & 0,340 & 0,149 & 0,191 & 0,022 & Confirmada \\
\hline H2 & DDR $\rightarrow$ DEF & $-1,116$ & 0,286 & $-0,495$ & 0,001 & Confirmada \\
\hline H3 & DDI $\rightarrow$ DEF & 0,915 & 0,046 & 0,337 & 0,001 & Confirmada \\
\hline H4 & Merc_Ext $\rightarrow$ DEF & 0,900 & 0,023 & 0,552 & 0,001 & Confirmada \\
\hline
\end{tabular}

Fonte: elaborado pelos autores (2019).

Conforme o resultado obtido nos testes de hipóteses é possível afirmar que todas as hipóteses propostas foram suportadas estatisticamente. A hipótese $\mathbf{H}_{\mathbf{1}}$ (a utilização das práticas de gestão estratégica de custos, dimensão melhoria dos processos (DMP), influencia positivamente no desempenho econômico-financeiro (DEF)) apresentou $\beta=0,191$ e o $\mathrm{p}$ valor $>0,05$. A hipótese $\mathbf{H}_{2}$ (A utilização das práticas de gestão estratégica de custos, dimensão redução de custos (DDR), influencia positivamente no desempenho econômico-financeiro (DEF)) apresentou $\beta=-0,495$ e o $\mathrm{p}$ valor $>0,05$. A hipótese $\mathbf{H}_{3}$ (a utilização das práticas de gestão estratégica de custos, dimensão desempenho em relação aos investimentos (DDI), influencia positivamente no desempenho econômico-financeiro (DEF)) que apresentou $\beta=0,337$ e o $\mathrm{p}$ valor $>0,05$. E por fim, a hipótese $\mathbf{H}_{4}$ (a atuação no mercado externo influencia positivamente no desempenho econômico-financeiro (DEF)) apresentou $\beta=0,552$ e o $\mathrm{p}$ valor $>0,05$.

As confirmações destas hipóteses corroboram com a literatura relacionada ao tema. Pizzini (2006) identificou, nos hospitais dos Estados Unidos da América, que a capacidade do sistema de custo de fornecer dados detalhados é favoravelmente associada, pelos gestores, com medidas de desempenho.

Henri, Boiral e Roy (2006) concluíram que o gerenciamento dos custos influencia no desempenho financeiro das empresas. Por meio de um estudo com 319 empresas identificaram que quando os custos ambientais são detalhados é possível geri-los e consequentemente obter um melhor desempenho financeiro nas organizações.

Posteriormente Cadez e Guilding (2012) identificaram que as empresas que utilizavam práticas da GEC pontuaram que o seu desempenho (retorno sobre o investimento, participação de mercado e novos produtos) estavam acima da média das demais empresas pesquisadas.

Além disso, o resultado da pesquisa comprova empiricamente o que a literatura afirma sobre o tema, como por exemplo, Shank e Govindarajan (1997) que indicam que as informações da Gestão Estratégica de Custos devem ser utilizadas para desenvolver estratégias e assim obter uma vantagem competitiva e maiores resultados. Também com Baines e Langfield-Smith (2003) que asseguraram que, além da formulação da estratégia, as informações adequadas sobre Gestão Estratégica de Custos têm o potencial de contribuir para as tomadas de decisões e consequentemente com o aumento do desempenho da organização. Ainda, com Anderson (2006) que afirmou que é por meio de um dos objetivos da Gestão Estratégica de Custos, o alinhamento dos custos com as estratégias, que a organização consegue uma melhor contribuição para otimizar o desempenho e ter mais resultado. Dihel (2006) que constatou que o Controle Estratégico de Custos pode ser entendido no contexto de uma organização como apoio à estratégia e, com isto, contribui efetivamente para melhor desempenho e maior efetividade estratégica. 


\section{Conclusão}

A principal implicação deste estudo foi a confirmação da existência de relação entre a utilização das práticas da Gestão Estratégica de Custos e o desempenho econômico-financeiro. As três hipóteses de pesquisa referentes a esta relação: H1. "a utilização das práticas de gestão estratégica de custos, dimensão melhoria dos processos (DMP), influencia positivamente no desempenho econômico-financeiro (DEF)"; $\mathbf{H}_{2}$. "a utilização das práticas de gestão estratégica de custos, dimensão redução de custos (DDR), influencia positivamente no desempenho econômicofinanceiro (DEF)"; e, H3. "a utilização das práticas de gestão estratégica de custos, dimensão desempenho em relação aos investimentos (DDI), influencia positivamente no desempenho econômico-financeiro (DEF)", foram confirmadas.

Ainda contribui, confirmando, que a atuação no mercado externo também influencia positivamente no desempenho econômico-financeiro, confirmando-se $\mathbf{H}_{\mathbf{4}}$ "atuação no mercado externo influencia positivamente no desempenho econômico-financeiro (DEF)."

Esta pesquisa trouxe contribuições gerenciais, pois comprovou a existência da relação entre a utilização das práticas da Gestão Estratégia de Custos e o Desempenho Econômico-Financeiro. Como contribuição acadêmica, a pesquisa validou o modelo teórico por meio de testes estatísticos de confiabilidade e consistência interna, bem como análise multivariada dos dados.

O estudo apresenta como uma das limitações a generalização dos resultados, pois a aplicação desta survey que se limitou a região da Serra Gaúcha. Outra limitação foi o tamanho da amostra analisada que contou com 87 empresas de pequeno, médio e grande porte dos setores metalomecânico, automotivo e eletroeletrônico filiadas ao SIMECS. Assim, as conclusões desta pesquisa restringem-se à realidade das empresas pesquisadas dos setores em foco. Também se ressalva, por tratar-se de uma pesquisa quantitativa, esta não possibilitou um maior aprofundamento em relação às análises.

Dessa forma, esta pesquisa comprovou existir relação entre a utilização das práticas da Gestão Estratégia de Custos e o Desempenho Econômico-Financeiro. Como estudos futuros sugere-se que este instrumento de pesquisa seja aplicado em outras atividades econômicas para avaliar se esta relação continua presente. Também sugere-se ampliar a quantidade de práticas da GEC no instrumento de coleta de dados, ampliando o alcance desta teoria. Por fim, sugere-se um estudo com método qualitativo para analisar em profundidade a utilização das práticas da Gestão Estratégica de Custos em organizações participantes deste estudo e que possuam elevado grau de utilização destas práticas, para que, dessa forma, os resultados possam auxiliar na aplicação das práticas da GEC por organizações que se interessem pelo tema.

\section{Referências}

ALMEIDA, F. A. S. Influência das políticas ambientais no desempenho empresarial econômico e socioambiental: um estudo do setor de leite e derivados de Goiás. 2010. 205 f. Tese (Doutorado em Administração) - Faculdade de Economia, Administração e Contabilidade da Universidade de São Paulo, São Paulo, 2010.

ALOTAIBI, M. B. G.; ZHANG, Y. The relationship between export market orientation and export performance: an empirical study. Applied Economics, v. 49, n. 23, p. 2253-2258, 2016. DOI: https://doi.org/10.1080/00036846.2016.1237743.

ALVES, F. P. A relação entre o grau de sofisticação na Gestão Estratégica de Custos na área de compras e o desempenho financeiro nas empresas brasileiras. 2012. 117 f. Dissertação (Mestrado em Administração). Escola de Negócios da Universidade Federal do Rio de Janeiro, Rio de Janeiro, 2012. 
ANDERSON, S. W. Managing costs and cost structure throughout the value chain: research on strategic cost management. Handbooks of Management Accounting Research, v. 2, p. 481-506, 2006. DOI: https://doi.org/10.1016/S1751-3243(06)02001-3.

ASSAF NETO, A. Estrutura e análise de balanços: um enfoque econômico-financeiro. São Paulo: Atlas, 2015.

ATKINSON, A. A.; BANKER, R. D.; KAPLAN, R. S.; YOUNG, S. M. Contabilidade gerencial. São Paulo: Atlas, 2000.

AZEREDO, A. J.; DE SOUZA, M. A.; MACHADO, D. G. Desempenho econômico-financeiro de indústrias calçadistas brasileiras: uma análise do período de 2000 a 2006. Revista de $\begin{array}{lllllllll}\text { Contabilidade e Organizações, v. } & 3, & \text { n. } & 6, & \text { p. } & 117-142, & 2009 . & \text { DOI: }\end{array}$ https://doi.org/10.11606/rco.v3i6.34744.

BAINES, A.; LANGFIELD-SMITH, K. Antecedents to management accounting change: a structural equation approach. Accounting, organizations and society, v. 28, n. 7-8, p. 675-698, 2003. DOI: https://doi.org/10.1016/S0361-3682(02)00102-2.

BARTZ, Daniel; STAUDT, Tarcisio; SOUZA, Marcos Antonio de. Gestão estratégica de custos: uso da engenharia reversa na análise dos custos de concorrentes. Revista de Administração e Contabilidade da Unisinos - BASE, v. 2, n. 3, p. 167- 175, 2005.

BENTLER, P. M. EQS structural equations program manual. Encino: Multivariate Software, 1995.

BYRNE, B. M. Structural equation modeling with AMOS: basic concepts, applications, and programming. New York: Taylor and Francis Group, 2010. DOI: https://doi.org/10.4324/9780203807644.

BOGDANOIU, C. Improving management accounting and cost calculation in dairy industry using standard cost method. Annals of the Constantin Brâncusi. University of Târgu Jiu, Economy Series, v. 2, p. 119-114, 2013.

CADEZ, S.; GUILDING, C. Benchmarking the incidence of strategic management accounting in Slovenia. Journal of Accounting \& Organizational Change, v. 3, n. 2, p. 126-146, 2007. DOI: 10.1108/18325910710756140.

CADEZ, S.; GUILDING, C. An exploratory investigation of an integrated contingency model of strategic management accounting. Accounting, organizations and society, v. 33, n7-8, p. 836863, 2008. DOI: https://doi.org/10.1016/j.aos.2008.01.003.

CADEZ, S.; GUILDING, C. Strategy, strategic management accounting and performance: a configurational analysis. Industrial Management \& Data Systems, v. 112, n. 3, p. 484-501, 2012. DOI: https://doi.org/10.1108/02635571211210086.

CHOW, C. W.; COOPER, J.; HADDAD, K. The effects of pay schemes and ratchets on budgetary slack and performance: A multiperiod experiment. Accounting, Organizations and Society, Elsevier, v. 16, n. 1, p. 47-60, 1991. 
COKINS, G. Measuring costs across the supply chain. Cost Engineering, v. 43, n. 10, p. 25-31, 2001.

DIEHL, C. A. Proposta de um sistema de avaliação de custos intangíveis. 1997. 128 f. Dissertação (Mestrado em Engenharia de Produção) - Programa de Pós-Graduação em Engenharia da Produção da Universidade Federal do Rio Grande do Sul, Porto Alegre, RS, 1997.

DIEHL, Carlos Alberto. Gestão Estratégica de Custos: identificando o alinhamento estratégico em uma empresa de segurança. Contab. Vista \& Rev., v. 17, n. 1, p. 69-97, jan./mar. 2006.

ELLRAM, Lisa M.; SIFERD, Sue Perrott. Purchasing: The cornerstone of the total cost of ownership concept. Journal of Business Logistics, v. 14, n. 1, p. 163-185, 1993.

FORNELL, C.; BOOKSTEIN, F. L. Two structural equation models: LISREL and PLS applied to consumer exit-voice theory. Journal of Marketing Research, 440-452, 1982. DOI: https://doi.org/10.1177\%2F002224378201900406.

FORNELL, C.; LARCKER, D. F. Evaluating structural equation models with unobservable variables and measurement error. Journal of Marketing Research, p. 39-50, 1981. DOI: https://doi.org/10.1177\%2F002224378101800104.

GIL, A. C. Métodos e técnicas de pesquisa social. 6ª ed. São Paulo: Atlas, 2008.

GITMAN, L. J. Princípios de administração financeira. 12. ed. São Paulo: Pearson Prentice Hall, 2010.

GOLAS, H.; MAZUR, A.; GRUSKA, J.; SZAFER, P. Application of the suggestion system in the improvement of the production process and product quality control. In: IOP Conference Series: Materials Science and Engineering. IOP Publishing. p. 06, 2016.

GUILDING, C.; CRAVENS, K. S.; TAYLES, M. An international comparison of strategic management accounting practices. Management Accounting Research, v. 11, n. 1, p. 113-135, 2000. DOI: https://doi.org/10.1006/mare.1999.0120.

HAIR Jr, J. F.; BLACK, W. C. B.; BARRY, J.; ANDERSON, R. E.; TATHAM, R. L. Análise multivariada de dados. Porto Alegre: Bookmann, 2009.

HAIR Jr., J. F.; BABIN, B.; MONEY, A. H.; SAMOUEL, P. Fundamentos de métodos de pesquisa em administração. Porto Alegre: Bookman, 2005.

HENRI, J. F.; BOIRAL, O.; ROY, M. J. Strategic cost management and performance: The case of environmental costs. The British Accounting Review, v. 48, n. 2, p. 269-282, 2016. DOI: https://doi.org/10.1016/j.bar.2015.01.001.

JOHNSON, H. T.; KAPLAN, R. S. Relevance Lost: the rise and fall of management accounting. Boston: HBSP, 1987.

JOHNSON, H. T.; KAPLAN, R. S. Contabilidade gerencial: a restauração da relevância da contabilidade nas empresas. Rio de Janeiro: Campus, 1993. 
JORESKOG, K. G.; SORBOM, D. Recent developments in structural equation modeling. Journal of Marketing Research, p. 404-416, $1993 . \quad$ DOI: https://doi.org/10.1177\%2F002224378201900402.

KAPLAN, R. S.; NORTON, D. P. Organização orientada para a estratégia. Rio de Janeiro: Campus, 2000.

KLINE, R. B. Principles and practice of structural equation modeling. 3 rd edition. New York: The Guilford Press, 2011.

KOTLER, Philip. Administração de marketing. 10. ed. São Paulo: Pearson, 2000. 764 p.

LANGFILED-SMITH, K. Strategic management accounting: how far have we come in 25 years? Accounting, Auditing \& Accountability Journal, v. 21, n. 2, p. 204-228, 2008. DOI: https://doi.org/10.1108/09513570810854400.

MARTINS, Eliseu. Contabilidade de Custos. São Paulo: Editora Atlas S.A. 2018. 408 p.

MARÔCO, J. Análise de equações estruturais: fundamentos teóricos, softwares \& aplicações. Lisboa: PSE, 2010.

MATITZ, Q. R. S.; BULGACOV, S. O conceito desempenho em estudos organizacionais e estratégia: um modelo de análise multidimensional. Revista de Administração Contemporânea, v. 15, n.4, p. 580-607, 2011. ISSN 1415-6555. http://dx.doi.org/10.1590/S141565552011000400003 .

NAKAGAWA, M. Gestão Estratégica de Custos: conceitos, sistemas e implementação. São Paulo: Atlas S.A, 1991.

NAUDÉ, W.; ROSSOUW, R. Export diversification and economic performance: evidence from Brazil, China, India and South Africa. Economic Change and Restructuring, v. 44, n. 1-2, p. 99134, 2011. DOI: https://doi.org/10.1007/s10644-010-9089-1.

PADOVEZE, C. L. Contabilidade de Custos: teoria, prática, integração com sistemas de informações (ERP). São Paulo: Cengage Learning, 2013.

PEREZ Jr., J. H; OLIVEIRA, L. M.; COSTA, R. G. Gestão estratégica de custos: testos, casos práticos e testes com as respostas. 8. edição. São Paulo: Atlas, 2012.

PEREZ Jr, J. H.; BEGALLI, G. A. A Elaboração das Demonstrações Contábeis. São Paulo: Atlas, 1999.

PIZZINI, M. J. The relation between cost-system design, managers' evaluations of the relevance and usefulness of cost data, and financial performance: an empirical study of US hospitals. Accounting, Organizations and Society, v. 31, n. 2, p. 179-210, 2006. DOI: https://doi.org/10.1016/j.aos.2004.11.001. 
RASIA, K. A. Práticas de Gestão Estratégica de Custos adotadas por empresas de segmentos do Agronegócio. 2011. 211 f. Dissertação (Mestrado em Ciências Contábeis) - Pós-Graduação em Ciências Contábeis da Universidade do Vale do Rio dos Sinos, São Leopoldo, 2011.

RIBEIRO, M. S. Contabilidade Ambiental. São Paulo: Saraiva, 2010. 240p.

ROCHA, W. Contribuição ao estudo de um modelo conceitual de sistema de informação de gestão estratégica. 1999. $158 \mathrm{f}$. Tese (Doutorado em Controladoria e Contabilidade). São Paulo: Universidade de São Paulo, 1999.

SAKURAI, M. Gerenciamento Integrado de Custos. São Paulo: Atlas S.A, 1997.

SHANK, J. K.; GOVINDARAJAN, V. A revolução dos custos: como reinventar e redefinir sua estratégia de custos para vencer em mercados crescentemente competitivos. Tradução Luiz Orlando Lemos. 2 ed. Rio de Janeiro: Campus, 1997.

SILVA, J. P. Administração Financeira. 7 ed. São Paulo: Atlas, 2005.

SIMECS. Sindicato das Indústrias Metalúrgicas, Mecânicas e de Material Elétrico de Caxias do Sul. Institucional. 2017. Disponível em: http://www.simecs.com.br/sindicato/institucional/. Acesso em: 22 out. 2017.

SIMMONDS, K. The Fundamentals of Strategic. Management Accounting, v. 15, n. 2, 1981.

SOUZA, M. A.; WEBER, E. L.; CAMPOS, R.H. Práticas de gestão de custos logísticos internos: estudo de caso em empresa moveleira do sul do Brasil. Revista Contemporânea de Contabilidade. Florianópolis, v. 12, n. 25, p. 27-46, 2015. DOI: http://dx.doi.org/10.5007/21758069.2015v12n25p27.

WRUBEL, F. Informações sobre Gestão Estratégica de Custos divulgadas por companhias abertas brasileiras. 2009. 145 f. Dissertação (Mestrado em Ciências Contábeis) - Pós-Graduação em Ciências Contábeis da Universidade do Vale do Rio dos Sinos, São Leopoldo, 2009. 